\title{
Synthesis of Molnupiravir
}

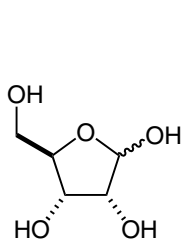

A

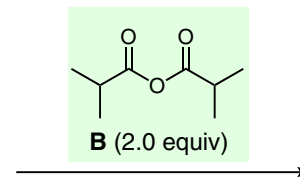

Novozyme 435 (10 wt\%) acetone $(1 \mathrm{~L}), 50^{\circ} \mathrm{C}, 12 \mathrm{~h}$ $92 \%$ assay yield $333 \mathrm{mmol}$ scale

* [ ] denotes a product used in the next step without further purification.

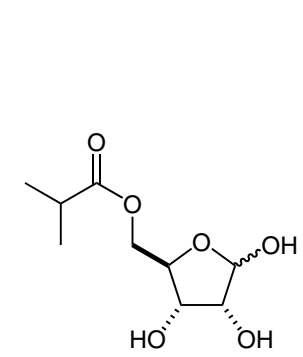

$[\mathrm{C}]^{\star}$

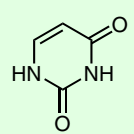

D $(272 \mathrm{mmol})$

C $(327 \mathrm{mmol})$

pyruvic acid (409 mmol)

MTR kinase $(3.6 \mathrm{~g})$

acetate kinase $(180 \mathrm{mg})$

pyruvate oxidase $(720 \mathrm{mg})$

catalase $(180 \mathrm{mg})$

uridine phosphorylase $(6.1 \mathrm{~g})$

aqueous buffer + cofactors

$$
\text { r.t., } 16 \mathrm{~h} \text {; }
$$

cryst ex EtOAc-heptane

$87 \%$ isolated yield
Synthesis of Natural

Products and

Potential Drugs

\section{Key words}

molnupiravir

nucleic acids

COVID-19

enzyme cascade

hexamethyldisilazane

dehydration

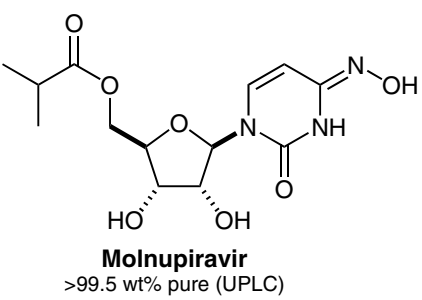
$\left(\mathrm{HO}-\mathrm{NH}_{2}\right)_{2} \cdot \mathrm{H}_{2} \mathrm{SO}_{4}(50 \mathrm{mmol})$ $\mathrm{NH}_{4} \mathrm{HSO}_{4}(100 \mathrm{mmol})$ imidazole $(20 \mathrm{mmol})$
HMDS (320 mmol) $80^{\circ} \mathrm{C}, 6 \mathrm{~h}$; cryst ex EtOAc-MTBE $86 \%$ isolated yield

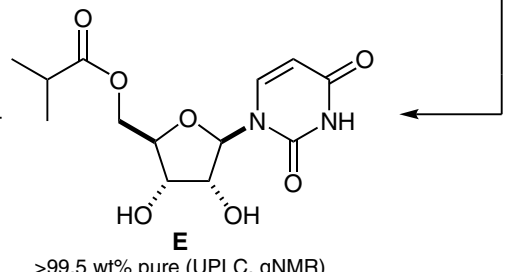

$>99.5 \mathrm{wt} \%$ pure (UPLC, qNMR)

Significance: Molnupiravir (MK-4482) is an orally available antiviral agent that reduces the risk of hospitalization or death from COVID-19 by $30 \%$ compared with placebo. The highly innovative enzymatic cascade that converts $\mathbf{C}$ to $\mathbf{E}$ comprises pyruvate oxidase and acetate kinase for ATP regeneration, MTR kinase (engineered) for the phosphorylation of $\mathbf{C}$, uridine phosphorylase (engineered) for uracil incorporation, and catalase to decompose hydrogen peroxide formed by pyruvate oxidase. This cascade can be run at high concentrations $(>80 \mathrm{~g} / \mathrm{L})$ of $\mathrm{C}$, forms the product in quantitative yield, and allows for easy isolation of crystalline $\mathbf{E}$ in $>99.5$ wt\% purity.
Comment: The conversion of $\mathbf{E}$ to molnupiravir occurs in nearly quantitative yield in neat HMDS. The initial product of the reaction is the bis-TMS derivative (not shown) that allowed easy removal of inorganic salts and byproducts via aqueous washes. Molnupiravir itself is highly water-soluble, and the removal of inorganic salts would be difficult without transient masking of the alcohols as TMS ethers. The TMS groups were easily cleaved by adjusting the $\mathrm{pH}$, after which the product crystallized. The synthesis depicted was accomplished in three steps and $69 \%$ overall yield from commodity chemicals. 